\title{
Validation of Operational WAVEWATCH III Wave Model Against Satellite Altimetry Data Over South West Indian Ocean Off-Coast of Tanzania
}

\author{
Chuki Sangalugeme ${ }^{1}$, Philbert Luhunga ${ }^{2}$, Agness Kijazi $^{2} \&$ Hamza Kabelwa $^{1}$ \\ ${ }^{1}$ Numerical Weather Prediction (NWP) Section, Division of Forecasting Services, Tanzania Meteorological \\ Agency, Dar es Salaam, Tanzania \\ ${ }^{2}$ Research Section, Division of Research and Applied Meteorology, Tanzania Meteorological Agency, Dar es \\ Salaam, Tanzania \\ Correspondence: Chuki Sangalugeme, Research Section, Division of Research and Applied Meteorology, \\ Tanzania Meteorological Agency, Dar es Salaam, Tanzania. E-mail: chuki.sangalugembe@gmail.com
}

Received: January 10, 2018

Accepted: January 15, 2018

Online Published: July 14, 2018

doi:10.5539/apr.v10n4p55

URL: https://doi.org/10.5539/apr.v10n4p55

\begin{abstract}
The WAVEWATCH III model is a third generation wave model and is commonly used for wave forecasting over different oceans. In this study, the performance of WAVEWATCH III to simulate Ocean wave characteristics (wavelengths, and wave heights (amplitudes)) over the western Indian Ocean in the Coast of East African countries was validated against satellite observation data. Simulated significant wave heights (SWH) and wavelengths over the South West Indian Ocean domain during the month of June 2014 was compared with satellite observation. Statistical measures of model performance that includes bias, Mean Error (ME), Root Mean Square Error (RMSE), Standard Deviation of error (SDE) and Correlation Coefficient (r) are used. It is found that in June 2014, when the WAVEWATCH III model was forced by wind data from the Global Forecasting System (GFS), simulated the wave heights over the Coast of East African countries with biases, Mean Error (ME), Root Mean Square Error (RMSE), Correlation Coefficient (r) and Standard Deviation of error (SDE) in the range of -0.25 to $-0.39 \mathrm{~m}, 0.71$ to $3.38 \mathrm{~m}, 0.84$ to $1.84 \mathrm{~m}, 0.55$ to 0.76 and 0.38 to 0.44 respectively. While, when the model was forced by wind data from the European Centre for Medium Range Weather Foresting (ECMWF) simulated wave height with biases, Mean Error (ME), Root Mean Square Error (RMSE), Correlation Coefficient (r) and Standard Deviation of error (SDE) in the range of -0.034 to $0.008 \mathrm{~m}, 0.0006$ to $0.049 \mathrm{~m}$, 0.026 to $0.22 \mathrm{~m}, 0.76$ to 0.89 and 0.31 to 0.41 respectively. This implies that the WAVEWATCH III model performs better in simulating wave characteristics over the South West of Indian Ocean when forced by the boundary condition from ECMWF than from GFS.
\end{abstract}

Keywords: WAVEWATCH III model, Boundary condition, Wavelength and wave height

\section{Introduction}

Marine Meteorology and Oceanography occupy a global role, serving a wide range of users, from international shipping, fishing and other met-ocean activities on the high seas, to the various activities which take place in coastal and offshore areas and on the coast itself. Having many people residing along the coastlines of South West Indian Ocean, information on the state of the ocean surrounding the East African countries is vital for the well being of the countrymen as well as for the socioeconomic development of these countries. The marine activities in East African countries range from conventional fishing to high-tech oil and natural gas exploration; transportation of goods to search and rescue operations in the high seas. Prior information of the state of the ocean would highly benefit these activities and ensure the safety of all those who venture into the sea. Furthermore, the ocean plays critical role in regulating the regional climate. In short, forecasting oceanographic parameters (both in surface and subsurface) at different time scales is extremely important for a wide spectrum of users ranging from weathermen to fishermen and from the navy to the offshore industries. Transportation by water is generally the most economical and efficient means to move goods. Issuing marine forecasts helps to reduce or avoid hazardous weather associated with wave height, swells and sea waves which are caused by strong winds in the oceans. More than 90 percent of the goods imported into the most of East African countries arrive via the oceans. Maritime operations commercial results in a 
main income contribution of millions of US dollars annually and generates thousand jobs in East African countries. The Ocean data including observations from Buoys are very scarce over the West Indian Ocean domain. The availability of these observations together with satellite observations will help improve the accuracy of the Ocean models used to issue marine forecast and warning over the region. The objective of this study is to validate WAVEWATCH III ${ }^{\circledR}$ over South West Indian Ocean (East African coast) so as to improve the marine forecasts issued by Meteorological centres in East Africa.

\section{Study Area}

The study area or domain is the South Western Indian Ocean (Coast of East Africa) filtered at Longitude $35^{\circ} 65^{\circ}$ and Latitude $-15^{0} 5^{0}$

South West Indian Ocean

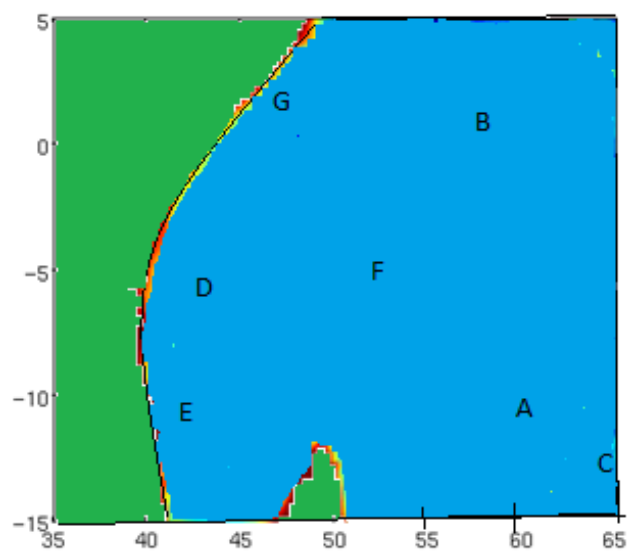

Figure 1. Indicate the study domain of South West Indian Ocean (SWIO) with the bathymetry information

\subsection{WAVEWATCH III® Model Descriptions}

WAVEWATCH III ${ }^{\circledR}$ (Tolman 1997, 1999a, 2009) is a third generation wave model developed at NOAA/NCEP in the spirit of the WAM model (WAMDIG 1988, Komen et al. 1994. WAVEWATCH III ${ }^{\circledR}$, however, differs from its predecessors in many important points such as the governing equations, the model structure, the numerical methods and the physical parameterizations. WAVEWATCH III ${ }^{\circledR}$ solves the random phase spectral action density balance equation for wave number-direction spectra. The implicit assumption of this equation is that properties of medium (water depth and current) as well as the wave field itself vary on time and space scales that are much larger than the variation scales of a single wave.

The spectral action balance equation is given by

$$
\begin{aligned}
& \frac{\partial N}{\partial t}+\nabla_{x} \cdot \dot{X} N+\frac{\partial \dot{k}}{\partial k} N+\frac{\partial \dot{\theta}}{\partial \theta} N=\frac{S}{\delta} \\
& X=c_{g}+U, \dot{k}=-\frac{\partial \delta}{\partial d} \frac{\partial d}{\partial s}-k \cdot \frac{\partial U}{\partial s} \\
& \dot{\theta}=-\frac{1}{k}\left[\frac{\partial \delta}{\partial d} \frac{\partial d}{\partial m}-k \cdot \frac{\partial U}{\partial m}\right]
\end{aligned}
$$

Where $C_{g}$ is group velocity, $k$ is the wave number, $\theta$ is the direction, $\delta=2 \pi f, s$ is a coordinate in the direction $\theta$ and $m$ is coordinate perpendicular to $s$

$$
S=S_{\mathrm{ln}}+S_{i n}+S_{n l}+S_{d s}+S_{b o t}+S_{a b}+S_{t r}+S_{s c}+S_{x x}
$$

Where $S_{l n}$ is the linear input term, $S_{i n}$ is the wind-wave interaction term, $S_{n l}$ is non-linear wave-wave interaction term, $S_{d s}$ is a dissipation (white-capping) term, $S_{b o t}$ is wave-bottom interaction term, $S_{d b}$ is depth induced breaking term, $S_{t r}$ is triad wave-wave interaction term, $S_{s c}$ is scattering of wave by bottom features, $S_{x x}$ is addition, user defined source term. 
Wave-induced current is the major driving force for near-shore circulation and sediment transport in the surf zone and inner continental shelf (Wright et al., 1991).

In shallow water, refraction and shoaling induce variations in the wave height. The most important physical processes which affect the waves in shallow waters are wave energy dissipation due to bottom friction (Shemdin et al., 1980), bottom induced wave breaking (Battjes \& Janssen, 1978) and wave-wave interaction (Madsen \& Sorensen, 1993). Interaction of waves with bottom produces a boundary layer, which results in the loss of wave energy to the bed due to bottom friction (Bagnold, 1946).

Vortex ripples and their feedback on the waves through enhanced bottom roughness determine the dissipation of wave energy in the bottom boundary layer (Zhukovets, 1963). Dissipation due to bottom friction is the primary wave attenuation mechanism in swell dominated conditions over a wide continental shelf (Ardhuin et al., 2003).

Waves along the west coast of India (WCI) are dominated by swells during southwest (SW) and northeast (NE) monsoon seasons and by wind seas during pre-monsoon season (Vethamony et al., 2011; Kumar et al., 2000; Rao and Baba, 1996). Kurian and Baba (1987) showed the importance of shelf slope in controlling spatial contrasts in bottom frictional attenuation and consequently the coastal energy regime. Wave heights along the WCI are generally low during NE and pre-monsoon seasons, and higher during SW monsoon (Aboobacker et al., 2011; Vethamony et al., 2009; Kumar and Kumar, 2008). Waves along the WCI are generally multi-peaked (Kumar et al., 2003), which is due to co-existence of swell and wind sea (Vethamony et al., 2009). The role of winds, in transforming the properties of swells, is not fully understood. However, wind seas are modified by the swells, and wind sea slope is preserved during their interactions (Hansen and Phillips, 1999). Aligned swells can shorten and attenuate the wind seas (Chu et al., 1992). The present study aims at understanding diurnal variation in wave parameters and wave transformation at various locations along the WCI and modification of wave parameters due to opposing/aligned winds and wind seas.

\section{Data and Methods}

\subsection{Data}

In this study surface winds forecast has been used (as initial conditions) from the Global Forecasting System (GFS) $\left(0.5^{0} \times 0.5^{0}\right)$ Global model and European Centre for Medium Range Weather Forecasting (ECMWF) $\left(0.25^{\circ} \times 0.25^{\circ}\right)$ model for the month of June 2014 data set. The high resolution reference bathymetry data (ETOPO1 and ETOPO2) from the National Geophysical Data Centre has also been used. Due to lack of observation data over South West Indian Ocean domain, only the satellite data which was downloaded from AVISO website (ftp://ftp.aviso.oceanobs.com/pub/oceano/AVISO/SSH/monomission/dt/corssh/ref/j2/) was used for wave model validation.

\subsection{Methodology}

The WAVEWATCHIII ${ }^{\circledR}$ wave model was set to simulate wave characteristics over different points over South West Indian Ocean domain for the period of one month. The evaluation of simulated wave characteristics against observations in the South West Indian Ocean domain is a difficult due to lack of conventional wave data from buoys and weather ships (in situ measurements). Recent advances in satellite technology have created a possibility to use remotely sensed wave data for wave model validation. In this study satellite data sets were used to validate the simulated wave parameters. Statistical methods for evaluation of model performance, such as bias, correlation analysis, Mean Square Error and Root Mean Square Error was used for the analysis.. The Grid Analysis and Display System (GrADS) and FERRET software were used for display of winds from models (ECMWF and GFS) as well simulation of wave products and risk maps. MatLab software has been used for statistical analysis

\section{Results}

Surface winds from European Centre for Medium Range Weather Forecasting (ECMWF) model were processed and displayed using ferret software to show the performance of the model. Plots below show the surface wind simulation from the ECMWF -reanalysis. 

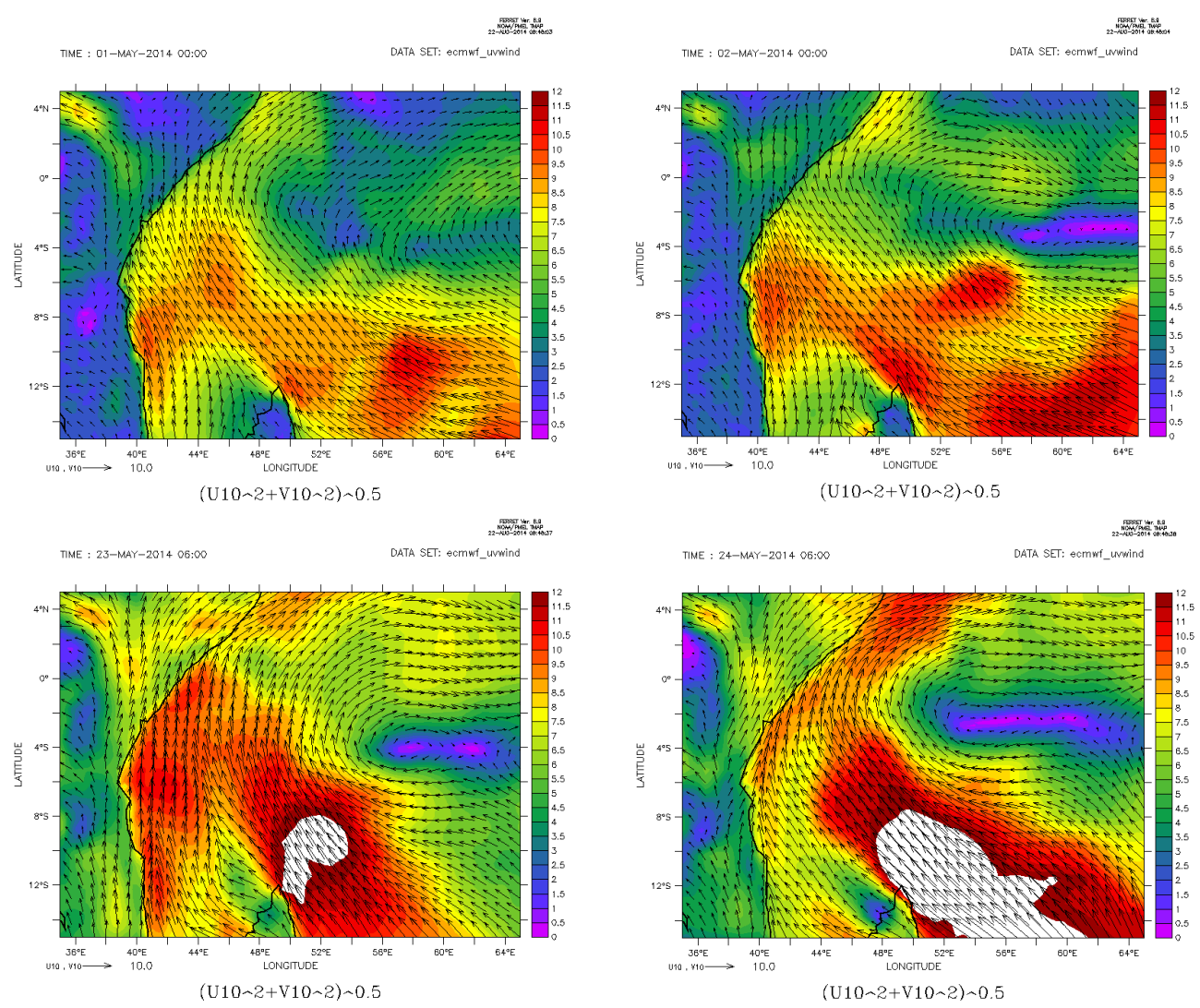

Comparison of winds from European Centre for Medium Range Weather Forecasting (ECMWF) and winds from satellite was done to observe if the forecast winds from ECMWF model matches with satellite wind data.

The figure below shows the plots of ECMWF winds and satellite.
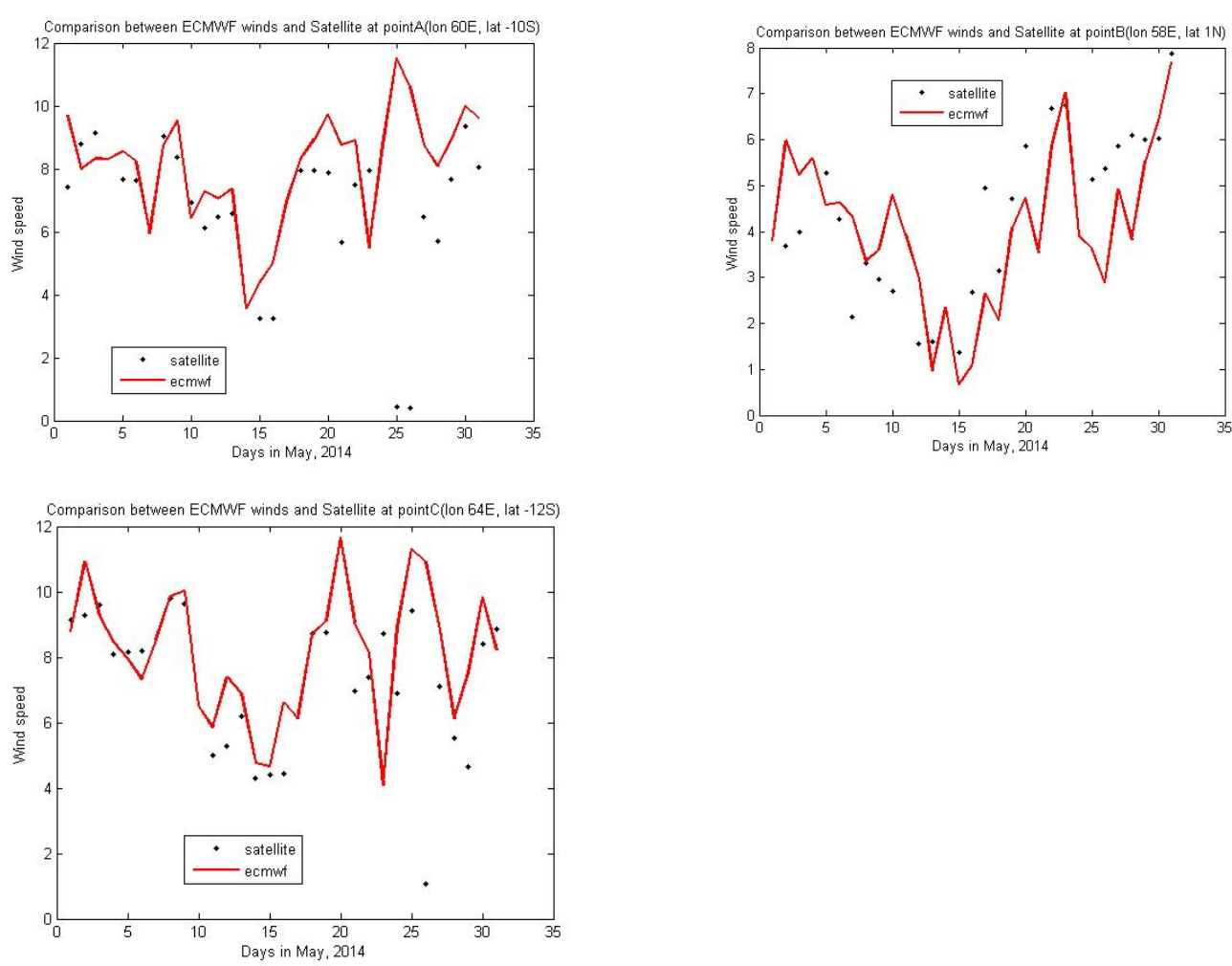

Figure 2. Comparison between satellite winds and ECMWF modelled winds 
From the plots of ECMWF winds with satellite data indicate that the ECMWF model forecasts almost matches with satellite winds despite of some gaps of wind data from satellite. These results give the clue of using winds data from ECMWF to the WAVE WATCH III wave model simulation.

The WAVE WATCH III model was set up for the period of one month (June 2014) using 10m wind forecast from the Global Forecasting System (GFS) model and European Centre for Medium Range Weather Forecasting (ECMWF) model. The results from the simulation of three day forecast of WAVE WATCH III WAVE MODEL which was forced by $10 \mathrm{~m}$ winds and surface air temperature from GFS is shown in the following figures below.
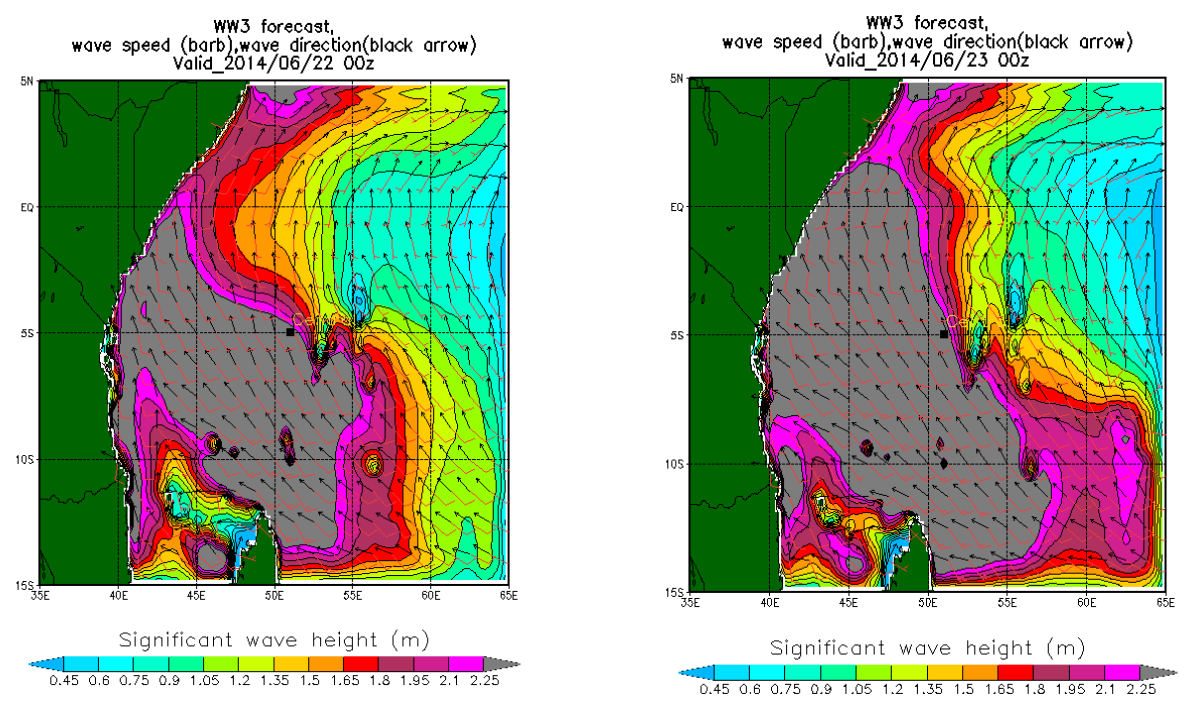

Figure 3. WAVE WATCH III WAVE MODEL forecast of 22/06/2014 and 23/06/2014 valid 00UTC for wave speed, wave direction and significant wave parameters
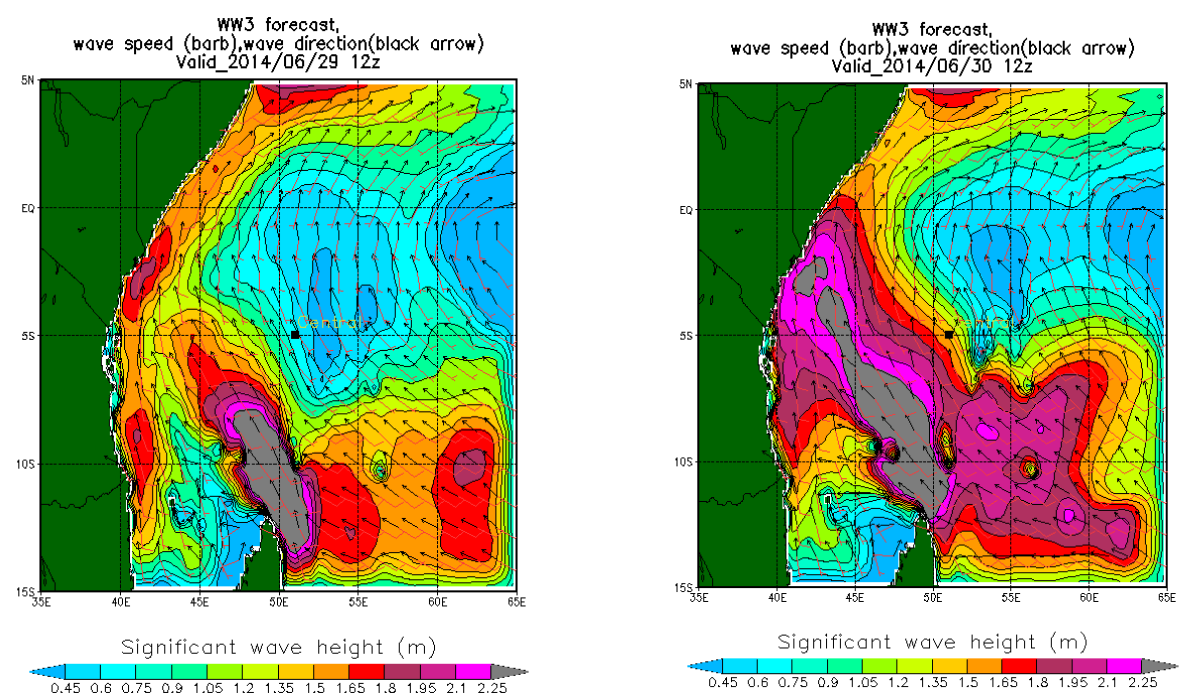

Figure 4. WAVE WATCH III WAVE MODEL forecast of 29/06/2014 and 30/06/2014 valid 12UTC for wave speed, wave direction and significant wave parameters (forced by winds and surface air temperature from GFS model

The simulation was done using winds and surface air temperature initial conditions from ECMWF model, the figure below are the forecast of WAVE WATCH III WAVE MODEL model. 


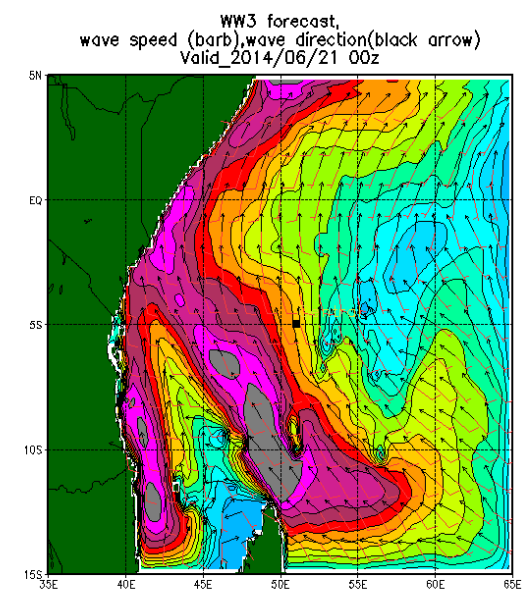

Significant wave height $(m)$

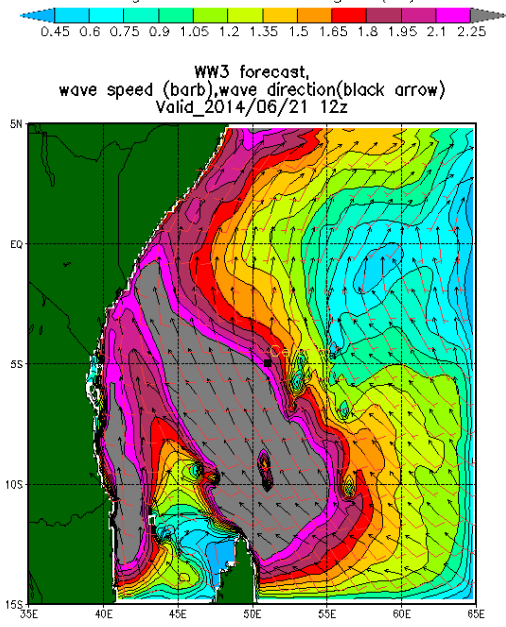

Significant wave height ( $m$ )

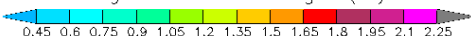

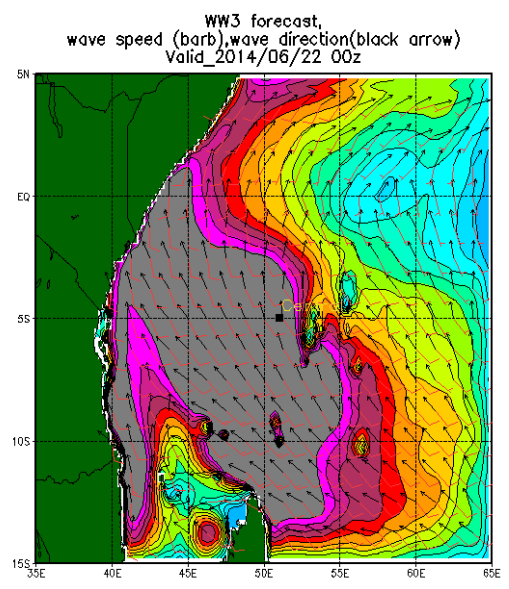

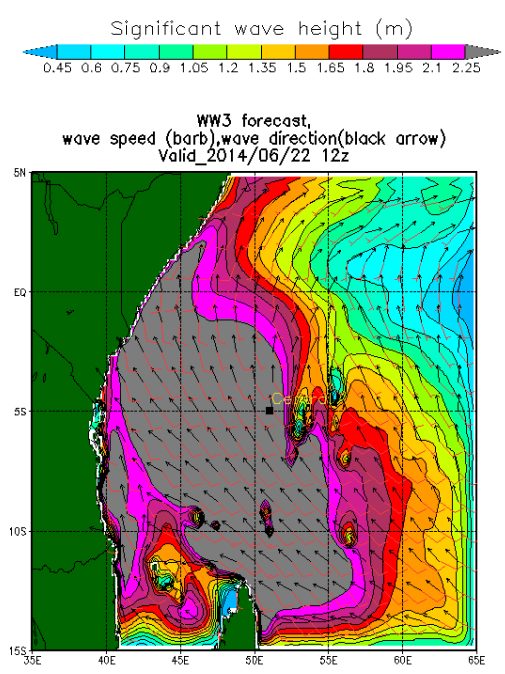

Significant wave height $(m)$

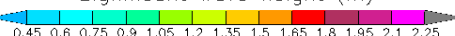

Figure 5. WAVE WATCH III WAVE MODEL forecast for wave speed, wave direction and significant wave parameters (forced by winds and surface air temperature from ECMWF model

The time series of Wave model simulations for the period of one month were plotted to observe the variation of model forecasts.

Figure $4 \mathrm{a}, \mathrm{b} \& \mathrm{c}$ show the hourly wave height simulation from the model for selected points over the SWIO.

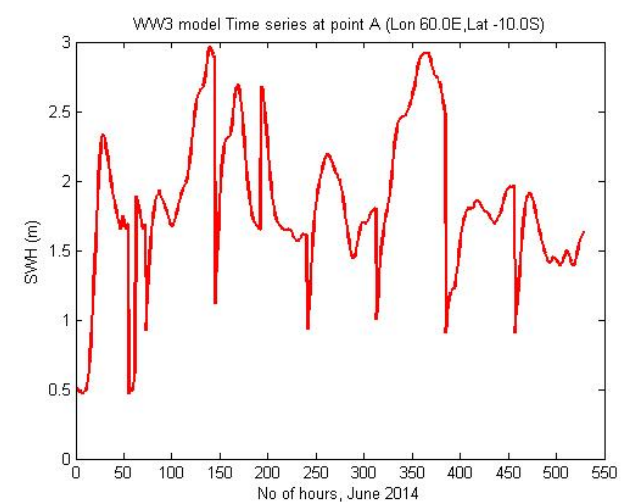

Figure 6(a) Wave model WAVE WATCH III WAVE MODEL Time series

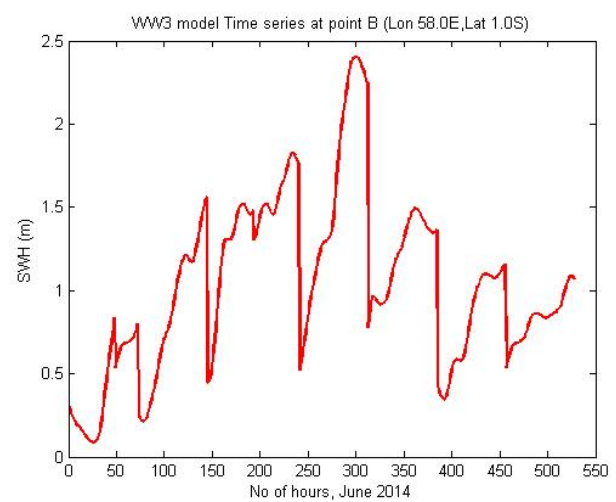

Figure 6(b) Wave model WAVE WATCH III WAVE MODEL Time series 




Figure 6(c) Wave model WAVE WATCH III WAVE MODEL Time series

Figure 6, 7 and 8 below show the plots of modelled significant wave heights (SWH) against satellite data where the WAVE WATCH III WAVE MODEL model was forced using winds from GFS model.

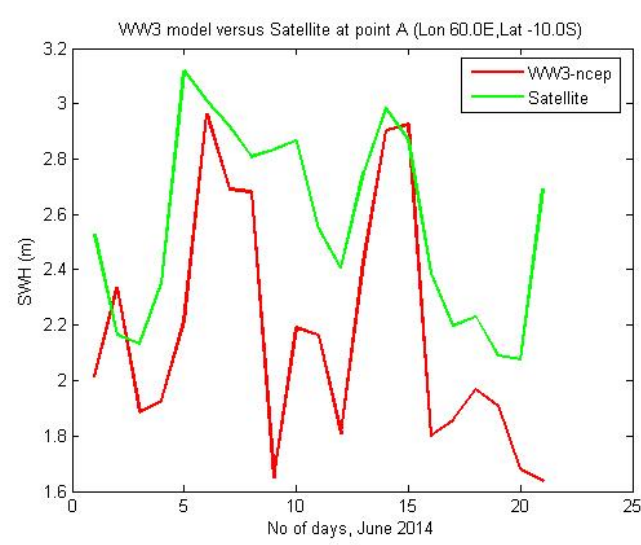

Figure 6. SWH simulation vs Satellite at point A

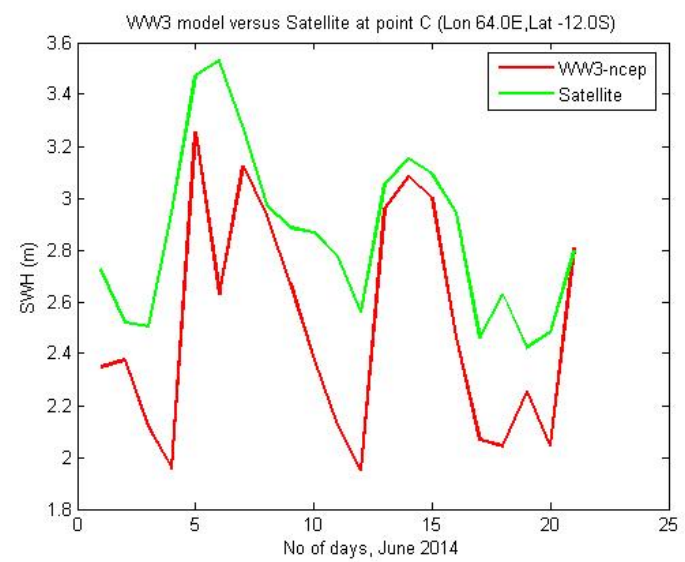

Figure 8. SWH simulation vs Satellite at point C

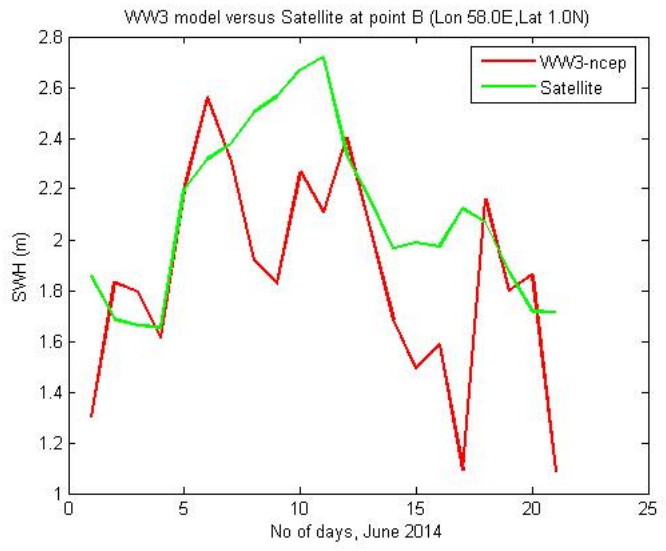

Figure 7. SWH simulation vs Satellite at point B

Figure 9, 10,11 and 12 below show the plots of modelled SWH against satellite data where the WAVE WATCH III WAVE MODEL model was forced using winds from ECMWF model. 


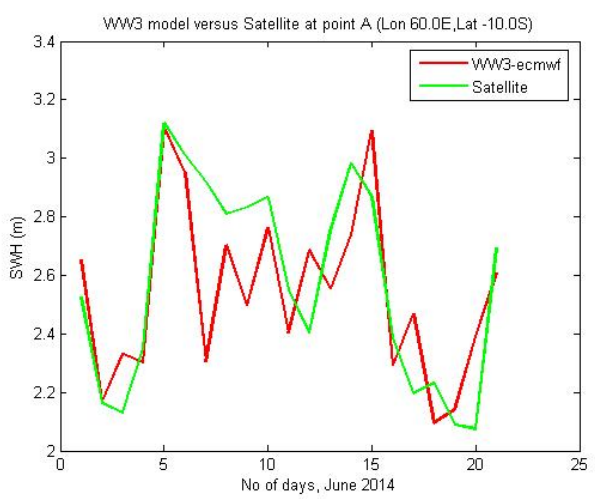

Figure 9. SWH simulation vs Satellite at point A

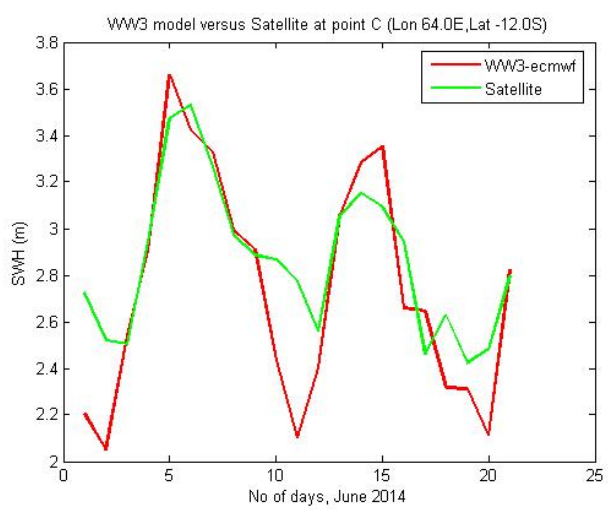

Figure 11. SWH simulation vs Satellite at point C

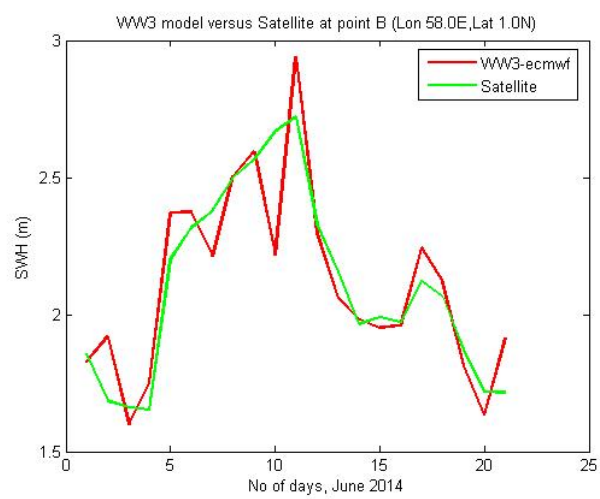

Figure 10. SWH simulation vs Satellite at poinB A

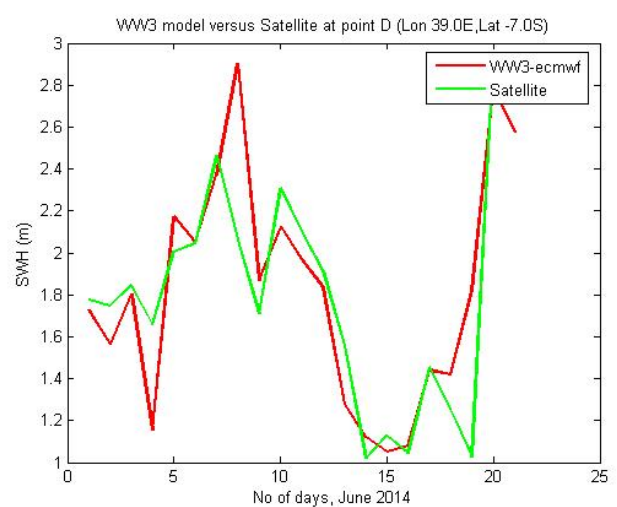

Figure 12. SWH simulation vs Satellite at point D

For the temporal and spatial matched WAVE WATCH III WAVE MODEL and satellite-altimeter data, comparison were undertaken by computing selected statistical variables (Bias, Mean Error, Root Mean Square Error (RMS), and correlation coefficient, r). The error is defined as:

Mean Error: $\quad M E=\frac{\sum_{i=1}^{N}\left(X_{i}-Y_{i}\right)^{2}}{N}$

Root Mean Square Error: ${ }_{R S M E}=\left(\frac{\sum_{i=1}^{N}\left(X_{i}-Y_{i}\right)^{2}}{N}\right)^{\frac{1}{2}}$

Standard deviation of error: $S D=\left[(R M S E)^{2}-(M E)^{2}\right]^{\frac{1}{2}}$

Correlation Coefficient: $\quad r=\frac{\sum_{i}^{N}\left(X_{i}-\overline{X_{i}}\right)\left(Y_{i}-\overline{Y_{i}}\right)}{\sqrt{\sum_{i}^{N}\left(X_{i}-\overline{X_{i}}\right)^{2}\left(Y_{i}-\overline{Y_{i}}\right)^{2}}}$

Where $X_{i}$ and $Y_{i}(\mathrm{i}=1,2,3, \ldots \mathrm{N})$ denote modelled and observed values respectively with $\bar{X}$ and $\bar{Y}$ their corresponding averaged values, $N$ is the number of collocated for comparison points. The Table 1 show statistical results of modelled SWH in respect to the satellite data 
Table 1. WAVE WATCH III WAVE MODEL modelled SWH (winds from GFS) versus Satellite Altimetry

Data for the period of $01-21$ June 2014

\begin{tabular}{llllll}
\hline Point Name & Bias $(\mathrm{m})$ & ME $(\mathrm{m})$ & RSME $(\mathrm{m})$ & Corr. Coefficient & Stdev $(\mathrm{m})$ \\
\hline A (Lon 60.0E, lat -10.0S) & -0.39 & 0.71 & 0.84 & 0.62 & 0.44 \\
B (Lon 58.0E, lat 1.0N) & -0.25 & 1.34 & 1.15 & 0.55 & 0.38 \\
C (Lon 64.0E, lat -12.0S) & -0.35 & 3.38 & 1.84 & 0.76 & 0.42 \\
\hline
\end{tabular}

Table 2. WAVE WATCH III WAVE MODEL model SWH (winds from ECMWF) versus Satellite Altimetry Data for the period of $01-21$ June 2014

\begin{tabular}{|l|l|l|l|l|l|}
\hline Point Name & Bias $(\mathrm{m})$ & ME (m) & RSME (m) & Corr. Coefficient & Stdev (m) \\
\hline A (Lon 60.0E, lat -10.0S & -0.034 & 0.049 & 0.22 & 0.76 & 0.31 \\
\hline B(Lon 58.0E, lat 1.0N & 0.008 & 0.022 & 0.148 & 0.898 & 0.33 \\
\hline C(Lon 64.0E, lat -12.0S & -0.12 & 0.0006 & 0.026 & 0.87 & 0.41 \\
\hline
\end{tabular}

The simulation of WAVE WATCH III WAVE MODEL wave model and its validation helped to generate the risk map that will be used as guidance to marine users' especially local and international shipping companies, industries, fisheries and other people living along the coast of Indian Ocean.

The figure below is the risk map generated.



Figure 11. Risk map for South West Indian Ocean

\section{Discussion}

The analysis for comparison between ECMWF modelled winds and satellite data showed that the model could be able reproduce the observed winds well. Figure 3 indicates that the WAVE WATCH III WAVE MODEL forecasted strong winds and large waves for very high significant $(>2.0 \mathrm{~m})$ almost over the entire coast of Mombasa in Kenya and Northern coast of Tanzania. However, on $29^{\text {th }}$ and $30^{\text {th }}$ of June 2014 at 12 UTC (Day Time), the WAVE WATCH III WAVE MODEL simulated waves over coast of Mombasa and Northern coast of Tanzania with no much significant signals in terms of strong winds and large waves. Strong wave characteristics was simulated over the deep ocean. Figure 6-8 indicate the comparisons of simulated and observed significante 
wave heights (SWH). It can be seen that the WAVE WATCH III WAVE MODEL forced by (winds from GFS) is slightly underestimating the values of SWH as observed by satellite altimetry data. While WAVE WATCH III WAVE MODEL model forced by (winds from ECMWF) (fig. 9- 12) show more improved accuracy in imulating the SWH as observed by satellite altimetry data.

The results from statistical analysis shows that in June 2014, when the WAVEWATCH III model was forced by wind data from the Global Forecasting System (GFS), simulated the wave heights over the Coast of East African countries with biases, Mean Error (ME), Root Mean Square Error (RMSE), Correlation Coefficient (r) and Standard Deviation of error (SDE) in the range of -0.25 to $-0.39 \mathrm{~m}, 0.71$ to $3.38 \mathrm{~m}, 0.84$ to $1.84 \mathrm{~m}, 0.55$ to 0.76 and 0.38 to 0.44 respectively. While, when the model was forced by wind data from the European Centre for Medium Range Weather Foresting (ECMWF) simulated wave height with biases, Mean Error (ME), Root Mean Square Error (RMSE), Correlation Coefficient ( $\mathrm{r}$ ) and Standard Deviation of error (SDE) in the range of -0.034 to $0.008 \mathrm{~m}, 0.0006$ to $0.049 \mathrm{~m}, 0.026$ to $0.22 \mathrm{~m}, 0.76$ to 0.89 and 0.31 to 0.41 respectively. This implies that the WAVEWATCH III model performs better in simulating wave characteristics over the South West of Indian Ocean when forced by the boundary condition from ECMWF than from GFS.

\section{Conclusion}

The conclusion that can be derived from this study is that the WAVE WATCH III WAVE MODEL wave model can provide better numerical simulations of wave conditions over the South West Indian Ocean and therefore could be used as guidance for marine weather forecast over the area as well as help to identify area of strong winds and large waves over Indian coast of East Africa. Moreover it is found that WAVE WATCH III WAVE MODEL simulation underestimates the Significate Wave Heights (SWH) over the South West Indian Ocean domain, Simulated SWH is more underestimated when WAVE WATCH III model is forced by winds from GFS than when it is forced by winds from ECMWF. The presented results may be used by ships for early planning routes and other people on beaches. The specific communities that will benefit directly are fisheries, tourists and people live along the coastline. It is recommended in this study to continue validation of WAVE WATCH III WAVE MODEL using long time data. This will help to give a reliable marine weather forecast and early warnings for severely events that are always happening over coastal areas

\section{References}

Aboobacker, V. M., Vethamony, P., \& Rashmi, R. (2011). "Shamal" swells in the Arabian Sea and their influence along the west coast of India. Geophys. Res. Lett., 38, $1-7$.

Alves, J. H. G. (2006). Numerical modelling of ocean swells contributions to the global wind-wave climate. Ocean Model., 11, 98-122.

Barber, N. F., \& Ursell, F. (1948). The generation and propagation of ocean waves and swell. I. Wave periods and velocities. Phil. Trans. R. Soc. Lond. A, 240(824), 527-560.

Battjes, J. A. \& Janssen, J. P. F. M. (1978). Energy loss and set-up due to breaking in random waves (pp. 569-587). Proc. 16th Coastal Engineering Conference, Hamburg, Germany.

Booij, N. R. R. C., Ris, R. C., \& Holthuijsen, L. H. (1999). A third-generation wave model for coastal regions: 1. Model description and validation. Journal of geophysical research: Oceans, 104(C4), 7649-7666.

Chawla, A., \& Tolman, H. L. (2007). Automated grid generation for WAVEWATCH III. Technical Bulletin, 254.

Chawla, A., Spindler, D., \& Tolman, H. L. (2011, October). A thirty year wave hindcast using the latest NCEP Climate Forecast System Reanalysis winds. In Proc. 12th Int. Workshop on Wave Hindcasting and Forecasting.

Durrant, T. H., Greenslade, D. J., \& Simmonds, I. (2009). Validation of Jason-1 and Envisat remotely sensed wave heights. Journal of Atmospheric and Oceanic Technology, 26(1), 123-134.

Kortcheva, A., Dimitrova, M., \& Galabov, V. (2012). A wave prediction system for real time sea state forecasting in Black Sea. arXiv preprint arXiv:1212.2123.

Kumar, V. S., \& Kumar, K. A. (2008). Spectral characteristics of high shallow water waves. Ocean Engineering, 35(8-9), 900-911.

Rio, M. H., \& Hernandez, F. (2003). High-frequency response of wind-driven currents measured by drifting buoys and altimetry over the world ocean. Journal of Geophysical Research: Oceans, 108(C8), 3283-3301. 
Schulz, E. W., Kepert, J. D., \& Greenslade, D. J. (2007). An assessment of marine surface winds from the Australian Bureau of Meteorology numerical weather prediction systems. Weather and Forecasting, 22(3), 613-636.

Tolman, H. L (1998): Validation of NCEP's Ocean winds for the use in wind wave models, Global Atmos. Ocean Sys., 6, 243-268.

Tolman, H. L. (2009). User manual and system documentation of WAVEWATCH III TM version 3.14. Technical note, MMAB Contribution, 276, 220.

\section{Copyrights}

Copyright for this article is retained by the author(s), with first publication rights granted to the journal.

This is an open-access article distributed under the terms and conditions of the Creative Commons Attribution license (http://creativecommons.org/licenses/by/4.0/). 